\title{
Active Cores in Deep Fields
}

\author{
Günther Hasinger ${ }^{1}$ and Andreas Müller ${ }^{2}$ \\ Max-Planck-Institute for Extraterrestrial Physics, P.O. box 1312, D-85741 Garching, \\ Germany \\ ${ }^{1}$ email: ghasinger@mpe.mpg.de ${ }^{2}$ email: amueller@mpe.mpg.de
}

\begin{abstract}
Deep field observations are an essential tool to probe the cosmological evolution of galaxies. In this context, $\mathrm{X}$-ray deep fields provide information about some of the most energetic cosmological objects: active galactic nuclei (AGN). Astronomers are interested in detecting sufficient numbers of AGN to probe the accretion history at high redshift. This talk gives an overview of the knowledge resulting from a highly complete soft X-ray selected sample collected with ROSAT, XMM-Newton and Chandra deep fields. The principal outcome based on X-ray luminosity functions and space density evolution studies is that low-luminosity AGN evolve in a dramatically different way from high-luminosity AGN: The most luminous quasars perform at significantly earlier cosmic times and are most numerous in a unit volume at cosmological redshift $z \sim 2$. In contrast, low-luminosity AGN evolve later and their space density peaks at $z \sim 0.7$. This finding is also interpreted as an anti-hierarchical growth of supermassive black holes in the Universe. Comparing this with star formation rate history studies one concludes that supermassive black holes enter the cosmic stage before the bulk of the first stars. Therefore, first solutions of the so-called hen-egg problem are suggested. Finally, status developments and expectations of ongoing and future extended observations such as the $X M M-C O S M O S$ project are highlighted.
\end{abstract}

Keywords. surveys, galaxies: active, galaxies: evolution, galaxies: high-redshift, galaxies: luminosity function, mass function, galaxies: nuclei, galaxies: statistics, X-rays: diffuse background, X-rays: galaxies, X-rays: general.

\section{Introduction}

Deep X-ray surveys turned out to be valuable diagnostic tools to investigate the galaxy formation history and large scale structure of the Universe. The diffuse X-ray background $(\mathrm{XRB})$ at the sky is composed of discrete sources that have been almost resolved by deep ROSAT, ASCA, Chandra and XMM-Newton observations at photon energies in the $0.1-10 \mathrm{keV}$ band $\dagger$ (Hasinger et al. (1998), Mushotzky et al. (2000), Giacconi et al. (2001), Hasinger et al. (2001), Giacconi et al. (2002), Alexander et al. (2003), Worsley et al. (2004), Bauer et al. (2004)). The X-ray sources in deep field observations are mostly active galactic nuclei (AGN). This has been confirmed by optical identification programmes with the Keck (Schmidt et al. (1998), Lehmann et al. (2001), Barger et al. (2001), Barger et al. (2003)), the VLT (Fiore et al. (2003), Szokoly et al. (2004), Zheng et al. (2004), Mainieri et al. (2005)) and COMBO-17 (Wolf et al. (2004)). All AGN are captured in different evolutionary stages. Therefore, X-ray deep fields also probe the in vivo growth of supermassive black holes (SMBHs) that drive the AGN luminosities according to a widely accepted paradigm. Optical identification programmes provide cosmological redshifts of the X-ray sources. This can be done by spectroscopic or, especially at high redshifts, photometric methods (Zheng et al. (2004), Mainieri et al. (2005), Wolf

$\dagger$ However, it must be stated that at higher photon energies in the $5-10 \mathrm{keV}$ band there is still a lack of identification. 
et al. (2004)). Optical observations demonstrate that the number distributions in redshift space of all galaxy types peak around $z \simeq 0.7$. Further, the AGN sample can be classified into AGN type-1 (unabsorbed, unobscured) and AGN type-2 (absorbed, obscured) by using optical and/or X-ray methods. In the optical, AGN type-1 are defined as sources exhibiting broad Balmer emission lines due to the fact that the observer is able to view the core of the AGN from the outside. These features are lacking for AGN type-2. In X-rays, AGN type-1 show an unabsorbed X-ray spectrum whereas AGN type-2 have absorbed spectra around photon energies from $0.5-1 \mathrm{keV}$.

The paper is organised as follows: In Sec. 2 the soft X-ray selected sample is presented, Sec. 3 treats the method to analyse features and evolution of different AGN object classes by means of X-ray luminosity functions. Space density and luminosity density evolutions as other tools are presented in Sec. 4. The observations are interpreted as a scenario described in Sec. 5. In Sec. 6 the results are compared to observational data from other work. Finally, we conclude in Sec. 7 .

\section{The X-ray selected AGN type-1 sample}

A sample of about 1000 AGN type-1 is considered that has been merged from ROSAT, Chandra and XMM-Newton surveys. The sources are restricted to the $0.5-2 \mathrm{keV}$ band and cover five orders of magnitude in flux and six orders of magnitude in survey solid angle. This sample is with $95 \%$ highly complete allowing to construct luminosity functions over cosmological timescales with unprecedented accuracy. Details of the soft X-ray sample are presented in Hasinger (2004) (H04 hereafter; see Table 1 therein). The advantage of restricting the sample only to type- 1 AGN is that systematic uncertainties are excluded a priori. AGN type- 2 can introduce these uncertainties by the varying and typically unknown AGN absorption column densities. As demonstrated by Szokoly et al. (2004) the optical AGN classification scheme suffers from dilution effects of AGN excess light from stars in the host galaxy - especially at low X-ray luminosities and medium redshifts. Then, only X-ray AGN classification schemes as introduced by Schmidt et al. (1998) and Szokoly et al. (2004) can do the job. As anticipated AGN are the main contributors to the XRB accounting for a fraction of about $70-100 \%$. A review about AGN and their engines, SMBHs, can be found in the PhD thesis by Müller (2004). It was found that at faintest X-ray fluxes there are contributions by other population classes such as starburst and normal galaxies, see Hornschemeier et al. (2000), Hornschemeier et al. (2001).

\section{X-ray luminosity functions}

As elaborated in Hasinger et al. (2005) (HMS05 hereafter) the X-ray luminosity functions (XLFs) of the sample presented in Sec. 2 are deduced by the following two methods: The first ('binned') method is presented in Miyaji et al. (2000) and is based on a variant of the $1 / V_{a}$ method. In this approach, the binned luminosity function in a given redshift bin $z_{i}$ is derived by dividing the observed number $N_{\text {obs }}\left(L_{x}, z_{i}\right)$ by the corresponding volume to the redshift range and appropriate survey X-ray flux limits and solid angles. Each of the XLFs is fitted by an analytical function to determine the bias in this luminosity value caused by a gradient of the XLF across one bin. By means of the resulting analytical function one can predict $N_{\bmod }\left(L_{x}, z_{i}\right)$. The ratio $N_{\text {obs }} / N_{\text {mod }}$ serves as a factor to correct the XLF due to bias to first order.

The second ('unbinned') method is based on unbinned data. Individual $V_{\max }$ values from ROSAT Bright survey (RBS) sources are used to evaluate the zero-redshift luminosity 


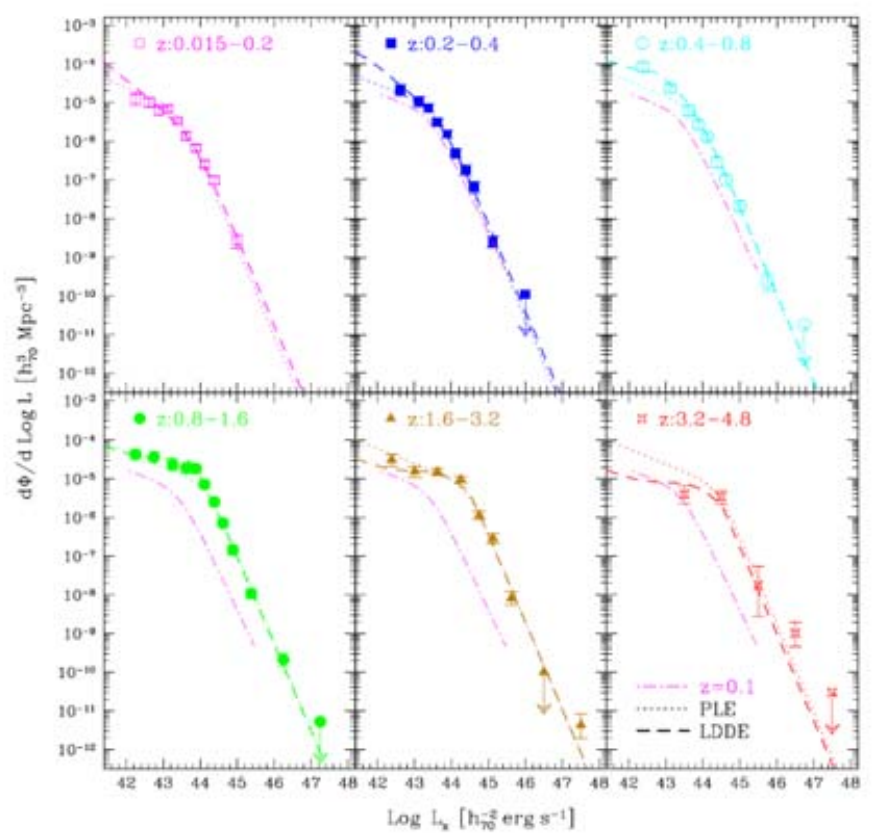

Figure 1. The soft X-ray luminosity function of the soft X-ray selected AGN type- 1 sample in different redshift shells as denoted. Error bars correspond to $68 \%$ Poisson errors of the AGN number in the bin. The best-fit two power-law model in the lowest $z$-shell $z=0.015-0.2$ is overplotted in each panel for reference. Dotted and dashed lines give best-fit PLE and LDDE models.

function (0zLF). By means of this LF the number of derived RBS sources matches accurately the observed number. Hence, the advantage of this method is that it is free from bias effects emerging in the first method. Evolution, i.e. space density as function of redshift, comes into play by introducing binning in luminosity and redshift. Again, bias effects are avoided by iterating the parameters of an analytical representation of the space density function. Together with the 0zLF this is used to predict $N_{\bmod }\left(L_{x}, z_{i}\right)$ for the surveys. Finally, the observed space densities in each bin are evaluated by multiplying by the ratio $N_{\text {obs }} / N_{\text {mod }}$ with the space density value. The result is shown in Fig. 1: This is the soft X-ray luminosity function (SXLF) in different redshift shells ranging from $z=0.015$ to $z=4.8$ of about 1000 AGN type- 1 . It clearly demonstrates that the LF shape varies with redshift. The typical two power-law model is confirmed. Fits of the $z$-dependent LF profiles strongly suggest a luminosity-dependent density evolution (LDDE) as outlined in HMS05. Pure luminosity evolution (PLE) overpredicts especially the LF behavior at high redshift, $z \sim 2-5$.

\section{Space density and luminosity density evolution}

An alternative plot to analyse the data is shown in Fig. 2: data are divided into luminosity classes and plotted over redshift. The plot clearly demonstrates the space density evolution for each luminosity class, i.e. compares low-luminosity AGN (LLAGN) to highluminosity AGN (HLAGN).The essential and surprising result is that the space density of HLAGN peaks at significantly higher redshift, $z \sim 2$, i.e. earlier cosmic times, than the LLAGN that peak at $z \sim 0.7$. In other words: Luminous quasars formed first and the bulk of faint AGN such as low-luminosity Seyferts came significantly later on the cosmic 


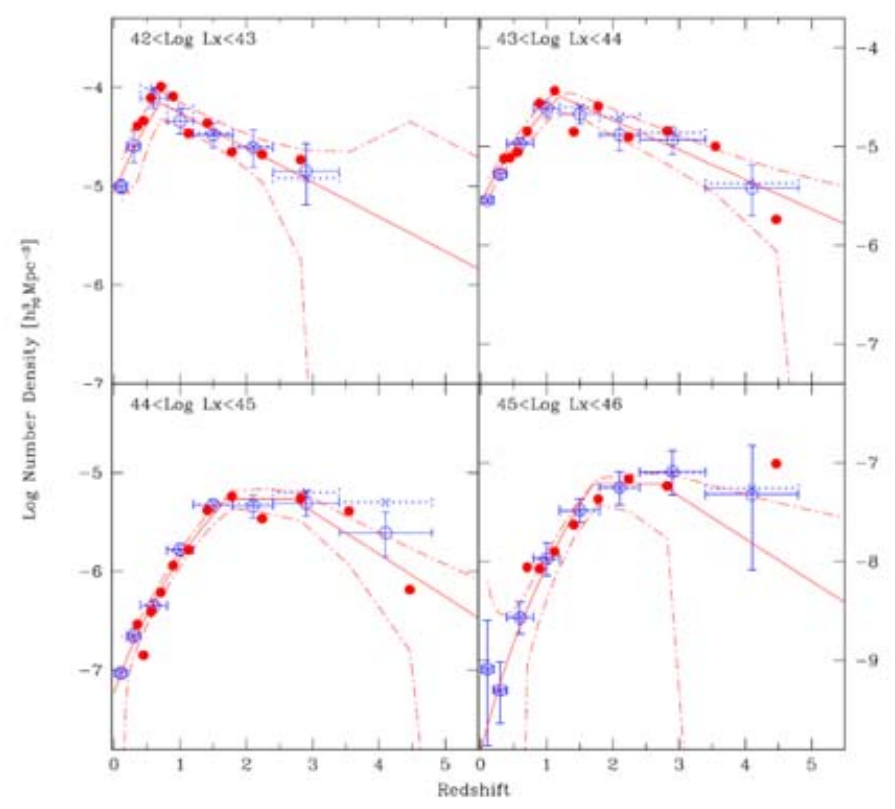

Figure 2. Space density evolution in different luminosity classes as derived from the binned (blue) and unbinnend method (red) outlined in Sec. 3. Dashed horizontal blue lines correspond to the maximum contribution of unidentified sources.

stage. Another remarkable fact is that for the first time the high-redshift decline in all luminosity classes with $\log \left(L_{X} /[\mathrm{erg} / \mathrm{s}]\right) \lesssim 45$ is shown. At higher luminositites insufficient statistics does not allow to derive secure trends. Space density evolution can also be studied using optically selected AGN type-1 as demonstrated by Croom et al. (2004) and Fan et al. (2001) using data sets from the $2 \mathrm{dF}$ and 6dF QSO redshift surveys. A comparison of an optical (UV) vs. an X-ray selection of AGN type-1 is elaborated in H04. The main result is that there is relatively little difference in space density evolutions between the two wave bands; however, we have to consider that (as demonstrated in Fig. 2) rise and fall of the space density is X-ray luminosity dependent. Therefore, comparisons of this kind are only preliminary and have to be re-analysed when larger samples of high-redshift X-ray selected QSOs are available.

A fruitful comparison is based on analysing flux correlations between optical and X-ray fluxes. The X-ray data from H04 and optical SDSS data from Vignali et al. (2003) are considered. Then, the X-ray flux in the soft $0.5-2 \mathrm{keV}$ band is plotted over optical $\mathrm{AB}_{2500}$ magnitudes. The first result is that the X-ray multi-cone survey covers a significantly wider range. Due to these better preconditions, a tight correlation was discovered by H04. Plotting the source's luminosity in the same filters indicates an essential difference: the luminosities in X-rays vs. those in redshifted $2500 \AA$ scale linearly, $L_{\mathrm{X}} \propto L_{\mathrm{UV}}$, when using X-ray selected data. But the correlation is non-linear, $L_{\mathrm{X}} \propto L_{\mathrm{UV}}^{0.75}$, when UV data are used. A preliminary interpretation of this discrepancy is sample selection effects. Host galaxy contamination corrections that are missing in the X-ray analysis are likely to be not responsible for the difference as $\alpha_{o x}$ value studies indicate (for details see H04). 


\section{Growth of supermassive black holes}

The analysis of X-ray deep field data by using XLFs and luminosity-weighted space density evolutions provides new clues for our understanding of AGN evolution in general and black hole growth in particular. AGN luminosity is connected to the central $\mathrm{SMBH}$ via Eddington's relation. Hence, from the observed activity the black hole mass can be estimated. Concerning HLAGN the soft X-ray selected samples clearly trace the rise and fall of quasars. The most luminous objects emerge firstly, grow rapidly by accretion and survive as supermassive dark remnants in the local Universe. Today, we observe these SMBHs in the centers of massive galaxy clusters such as M87 in the Virgo cluster. This scenario gets support from theoretical simulations presented recently by Springel et al. (2005): In hydrodynamical simulations of structure formation on large scales performed in a cube with $\approx 700 \mathrm{Mpc}$ size, the evolution of cold dark matter (CDM) halos are followed from $z \sim 120$ to $z \sim 0$. It has been shown that the observed large scale structure with superclusters surrounded by smaller clusters can be reproduced. Furthermore, the simulations strongly suggest that the most massive black holes with $10^{10} \mathrm{M}_{\odot} \dagger$ can be found in the centers of the most massive clusters. These are the descendants of the most active accretors in the quasar era at $z \sim 2$. X-ray deep field data from the soft band now suggest an anti-hierarchical growth of black holes: the most massive black holes with $10^{8}-10^{10} \mathrm{M}_{\odot}$ formed first and the bulk of the lighter black holes with $10^{6}-10^{8} \mathrm{M}_{\odot}$ formed later. This behaviour is totally unexpected and not predicted by standard CDM structure formation scenarios which are hierarchical. Maybe this observational fact hints for two accretion modes that differ in accretion efficiency (Duschl \& Strittmatter (2002)). However, a self-consistent model which explains both, anti-hierarchical black hole growth and the local black hole mass function derived from the $M_{\mathrm{BH}}-\sigma$ relation assuming two accretion modes has been suggested recently by Merloni (2004). Concretely, two accretion modes can be established by a varying value of efficiency $\epsilon$ - the parameter in accretion theory that controls conversion of mass flux into radiation flux. Black hole angular momentum controles crucially the efficiency: it is rather low, $\epsilon \sim 0.054$, for Schwarzschild black holes, or high, $\epsilon \sim 0.37$, for Kerr black holes as has been found by Thorne (1974). The SXLFs that give rise to an early quasar era in combination with the evolution of the star formation rate (SFR) also provide new clues to the so-called hen-egg problem i.e. if galaxies or the SMBHs came first in the Universe. The history of global cosmic star formation has been determined using Hubble deep field (HDF) observations (see Madau (1997), Connolly et al. (1997)) corrected for dust obscuration following Pettini et al. (1997). The resulting SFR as function of redshift shows only a moderate variation with cosmic time with a possible peak around a redshift of unity and a steep decline towards lower redshifts. But the AGN population reveals a pronounced peak at significantly higher redshifts as shown in the SXLF of AGN type-1. This discrepancy indicates that the bulk of SMBHs have been in place before the bulk of stars in these galaxies formed.

Very recently, an analysis of the specific SFR (SSFR) i.e. the star formation rate per unit stellar mass, has been presented based on FORS Deep Field (FDF) and GOODS-S field data, see Feulner et al. (2005). Their main finding is that the most massive galaxies with stellar masses around $10^{11} \mathrm{M}_{\odot}$ are in quiescent state for $z \lesssim 2$ but that the SSFR is increased by a factor of $\sim 10$ for redshifts $z \gtrsim 2$. This is interpreted as a very early formation epoch of most massive galaxies as supported by the Millennium simulation (Springel et al. (2005)) and X-ray deep field observations (HMS05). Comparisons of deep field observations therefore allow for attractive solutions of the hen-egg problem.

$\dagger$ and also the oldest population of stars (PopIII). 


\section{Discussion}

In this section the analysis with soft X-ray data of about 1000 AGN type-1 are compared to other work. Ueda et al. (2003) analysed the hard X-ray luminosity functions (HXLFs) of bright AGN using $A S C A$ data. This hard X-ray selected sample is highly complete ( $\sim 95 \%)$ and consists of both, AGN type- 1 and type-2, comprising $\approx 230$ sources. The main feature is that the fraction of type-2 AGN decreases with intrinsic luminosity or in other words: the space density of obscured AGN is luminosity-dependent. A possible explanation for this trend may be due to clean-out effects because the luminous AGN core may blow away the dusty torus at the pc-scale by radiation. Low-luminosity cores are not strong enough to initiate such a decay of the mass reservoir. Therefore, the AGN classification scheme into type- 1 and type- 2 is not only a pure orientation effect but is also dependent on AGN luminosity. As outlined in Sec. 3 the SXLFs strongly suggest a LDDE. Interestingly, the hard X-ray selected sample by Ueda et al. (2003) demonstrates the same behaviour. There is only a difference in the normalization by a factor of five probably due to absorbed objects missing in the SXLF. Another soft and hard X-ray selected sample based on Chandra $(C D F-N, C D F-S, C L A S X S)$ and $A S C A$ data has been presented recently by Barger et al. (2005). Their results are in good agreement with the SXLF analysis presented here, but they still suffer from substantial identification incompleteness. Their AGN type-1 sample does not directly compare to the one discussed here because Barger et al. (2005) only include optically classified AGN type-1 (broad-line AGN) but miss most of LLAGN type-1 that are considered here. A critical comparison of all available XLFs from different observations will be the topic of an upcoming paper. Recently it turned out that there is a possible new population of star-forming galaxies that can be found especially at very low fluxes, $S_{\mathrm{X}} \lesssim 10^{-16} \mathrm{erg} \mathrm{cm}^{-2} \mathrm{~s}^{-1}$ as discovered by Hornschemeier et al. (2000), Hornschemeier et al. (2001), Rosati et al. (2002), Norman et al. (2004). Upcoming X-ray selected samples will have to account for this special class by lowering the flux limits.

\section{Conclusions}

In general, X-ray deep fields prove valuable tools to investigate the formation and evolution history of galaxies, in particular of AGN. Concerning AGN, X-ray data sets cannot stand alone; they need additional support from optical identification programmes that discriminate type- 1 vs. type- 2 and deliver redshift determinations by spectroscopic or photometric techniques. Then, the analysis follows two branches: samples are constructed from soft and/or hard X-ray energy bands. To date soft X-ray selected samples provide high-quality data samples with high degrees of completeness. Hard X-ray selected samples still suffer from lacking spectroscopic identification. There is much work to be done in the future to resolve the high-energy XRB branch into discrete sources. Nevertheless, the comparison of the results from soft (HMS05) and soft plus hard X-ray selected samples by Ueda et al. (2003) and Barger et al. (2005) agrees well so far.

The principal method to analyse X-ray samples is based on deriving XLFs, space density evolution and luminosity density evolution. The SXLF sample presented here strongly suggests dramatically different evolutionary paths of LLAGN and HLAGN: Most luminous quasars formed significantly earlier as the space density peak at $z \approx 2$ demonstrates. The bulk of galaxies with low luminosities such as Seyferts perform later with a space density peak at $z \approx 0.7$. Linking AGN luminosiy to black hole mass via the Eddington criterion, one immediately arrives at the finding that the growth of SMBHs is antihierarchical: The most massive holes form first. A comparison with the SFR evolution 


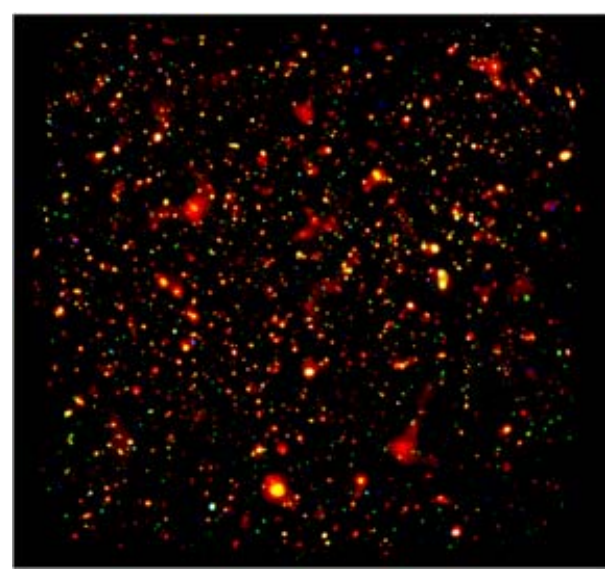

Figure 3. RGB image of the XMM-COSMOS deep field first year data. The 2 square degree field consists of 25 separate pointings amounting 1.4 Ms exposure time in total. Red colors belong to $0.5-2 \mathrm{keV}$ photon energies, green to $2-4.5 \mathrm{keV}$ and blue to $4.5-10 \mathrm{keV}$. Courtesy: Nico Cappelluti and Alexis Finoguenov (both MPE Garching)

even shows that the most massive holes formed before the bulk of first stars formed. Hence, deep field observation have the power to solve the cosmological hen-egg problem. The observed AGN evolution scenario should gain support from theoretical simulations. The Millennium simulation is one essential cornerstone that allows to predict the evolution of the large scale structure.

The next observational steps are to collect better data i.e. identify more sources, perform deeper pencil beams and adjust suitable flux limits. One upcoming multi-wavelength project is called COSMOS. This is a HST treasury project where a 2 square degree field is observed with $A C S$ by using $10 \%$ of observing time over two years. There are commitments from VLA, VLT, Subaru and XMM-Newton so that there will be also radio to X-ray data available for the same field where more than two million sources are supposed to be detected. Main goals of COSMOS are to study the large scale structure (LSS), evolution of galaxies, AGN and dark matter haloes, to investigate the SFR, and to derive AGN activity as function of morphology, size, redshift and LSS environment. One pencil beam with 0.2 square degree is included. The sensitivity level amounts $5 \times 10^{-16} \mathrm{erg} \mathrm{cm}^{-2} \mathrm{~s}^{-1}$ and captures therefore sources that are by a factor of two fainter than in deepest ROSAT surveys $\dagger$. First year observations of XMM-COSMOS started in December 2003. AO3 data are now available: A preliminary RGB image of 2 square degree size and 1.4 Ms total exposure time is shown in Fig. 3. As expected, a wealth of sources, almost all AGN, can be seen. The analysis of these XMM-COSMOS data is subject of a future paper (Hasinger et al. (2006)). Other upcoming extended deep X-ray surveys such as eRosita and eCDFS will improve significantly the analysis presented here.

\section{Acknowledgements}

AM and GH would like to thank the organizers of the IAU symposium No. 230 in Dublin, Ireland.

$\dagger$ Detailed information is provided on the COSMOS website http://www.astro.caltech. edu/ cosmos/ and the XMM-COSMOS website http://www.mpe.mpg.de/XMMCosmos 


\section{References}

Alexander, D.M., Bauer, F.E., Brandt, W.N., et al. 2003, AJ 126, 539

Barger, A.J., Cowie, L.L., Mushotzky, R.F., Richards, E.A., et al. 2001, AJ 121, 662

Barger, A.J., Cowie, L.L., Capak, P., et al. 2003, AJ 126, 632

Barger, A.J., Cowie, L.L., Mushotzky, R.F., et al. 2005, AJ 129, 578

Bauer, F.E., Alexander, D.M., Brandt, W.N., et al. 2004, AJ 128, 2048

Connolly, A.J., Szalay, A.S., Dickinson, M., et al. 1997, A\&A 319, 7

Croom, S.M., Smith, R.J., Boyle, B.J., et al. 2004, MNRAS 349, 1397

Duschl, W.J. \& Strittmatter, P.A. in Active Galactic Nuclei: from Central Engine to Host Galaxy Abstract Book, meeting held in Meudon, France, July 23-27, 2002, Eds.: S. Collin, F. Combes and I. Shlosman. To be published in ASP Conference Series, p. 76

Fan, X., Strauss, M.A., Schneider, D.P., et al. 2001, AJ 121, 54

Feulner, G., Gabasch, A., Salvato, M., et al. 2005, astro-ph/0509197, accepted for ApJL

Fiore, F., Brusa, M., Cocchia, F., et al. 2003, A\&A 409, 79

Giacconi, R., Rosati, P., Tozzi, P., et al. 2001, ApJ 551, 624

Giacconi, R., Zirm, A., Wang, J.X., et al. 2002, ApJS 139, 369

Hasinger, G., Burg, R., Giacconi, R., et al. 1998, A\&A 329, 482

Hasinger, G., Altieri, B., Arnaud, M., et al. 2001, A\&SA 365, 45

Hasinger, G. 2004, Nucl. Physics B. Suppl. Ser., 132, 86 (H04)

Hasinger, G., Miyaji, T., \& Schmidt, M. 2005, astro-ph/0506118, A\&A in press (HMS05)

Hasinger et al. 2006, ApJ, in prep.

Hornschemeier, A.E., Brandt, W.N., Garmire, G.P., et al. 2000, ApJ 541, 49

Hornschemeier, A.E., Brandt, W.N., Garmire, G.P., et al. 2001, ApJ 554, 742

Lehmann, I., Hasinger, G., Schmidt, M., et al. 2001, A\& A 371, 833

Madau, P. 1997, AIP Conf. Ser. 393, 481

Mainieri, V., Rosati, P., Tozzi, P., et al. 2005, A\&广A 437, 805

Merloni, A. 2004, MNRAS 353, 1035

Miyaji, T., Hasinger, G., \& Schmidt, M. 2000, A $\& A$ 353, 25

Müller, A. 2004, PhD thesis, Landessternwarte Heidelberg, Germany, Black Hole Astrophysics: Magnetohydrodynamics on the Kerr Geometry

Mushotzky, R.F., Cowie, L.L., Barger, A.J., \& Arnaud, K.A. 2000, Nature 404, 459

Norman, C., Ptak, A., Hornschemeier, A., et al. 2004, ApJ 607, 721

Pettini, M., Steidel, C.C., Adelberger, K.L., et al. 1997, astro-ph/9708117, To appear in ASP Conference Series ORIGINS, J.M. Shull, C.E. Woodward, and H. Thronson (eds.)

Rosati, P., Tozzi, P., Giacconi, R., et al. 2002, ApJ 566, 667

Schmidt, M., Hasinger, G., Gunn, J.E., et al. 1998, A\&A 329, 495

Springel, V., White, S.D.M., Jenkins, A., et al. 2005, Nature 435, 629

Szokoly, G., Bergeron, J., Hasinger, G., et al. 2004, ApJS 155, 271

Thorne, K.S., 1974, ApJ 191, 507

Ueda, Y., Akiyama, M., Ohta, K., \& Miyaji, T. 2003, Astron. Nachr. 324, 36

Ueda, Y., Akiyama, M., Ohta, K., \& Miyaji, T. 2003, ApJ 598, 886

Vignali, C., Brandt, W.N., \& Schneider, D.P. 2003, AJ 125, 433

Wolf, C., Meisenheimer, K., Kleinheinrich, M., et al. 2004, A\&SA 421, 913

Worsley, M., Fabian, A.C., Mateos, S., et al. 2004, MNRAS 352, L28

Zheng, W., Mikles, V. J., Mainieri, V., et al. 2004, ApJS 155, 73

\section{Discussion}

ELVIS: There has been concern that quite subtle selection effects can produce e.g. $\mathrm{z}=0.7$ peak and LLAGN detectability (Treister et al). What is your reaction to this study?

MÜLLER: I am sorry. I don’t know this particular paper and cannot comment on that. 